\title{
Assessing the Impact of Diabetes Mellitus and End Stage Renal Disease on Cervical Cancer Prognosis in an Urban Population
}

K. Cotangco ${ }^{1}$, A. Kaushiva², L. Pinkard¹, R. Arya ${ }^{3}$, J. Jutzy³ , K. Tossas Milligan², C. Son $^{3}$, R. Kothari ${ }^{4}$

${ }^{1}$ University of Illinois, Chicago, Obstetrics and Gynecology, ${ }^{2}$ University of Illinois, Chicago, Cancer Center

${ }^{3}$ University of Chicago, Radiation Oncology, ${ }^{4}$ University of Illinois, Chicago, Gynecologic Oncology

\section{Objectives}

Diabetes mellitus (DM) and end stage renal disease (ESRD) are medical comorbidities causing immunosuppression. The role of DM in cervical cancer prognosis is controversial; the role of ESRD has not been studied. We examined the prognostic effect of these immunosuppressive conditions on cervical cancer survival in an urban population. The effect of tobacco use on prognosis in these groups was also analyzed.

\section{Methods}

A retrospective analysis of women diagnosed with cervical cancer between 2005-2016 was performed. Patients were identified using the institution's tumor registry. Demographic information, disease stage, treatment, and comorbidities (BMI, smoking status, presence of $D M$, and ESRD were abstracted from the charts. DM and ESRD) were defined as immunosuppressive states. Baseline characteristics between immunosuppressed (IS) and nonimmunosuppressed ( $\mathrm{NI}$ ) patients were compared using Chi-square. Cox proportional hazards model was used to compare progression-free and overall survival.

\section{Results}

337 women were identified; 264 women were eligible for analysis. 9.2\% $(n=24)$ of patients were diabetic, $2 \% \quad(n=5)$ had ESRD. Baseline characteristics were similar between the IS and $\mathrm{NI}$ groups with the exception that IS patients had significantly higher BMI levels $(>30)$ $(p<0.001)$. Progression-free survival (PFS) was significantly worse amongst IS patients (HR 1.92, 95\% Cl 1.05-3.48). Overall survival (OS) approached statistical significance for IS patients $(H R=1.76$, $95 \% \mathrm{Cl}$ 0.97-3.2). In patients with DM or ESRD, smoking tobacco did not affect PFS or OS.

\section{Conclusions}

PFS in cervical cancer patients is affected by DM and ESRD. The effect on OS approached statistical significance. Smoking tobacco did not affect survival. 